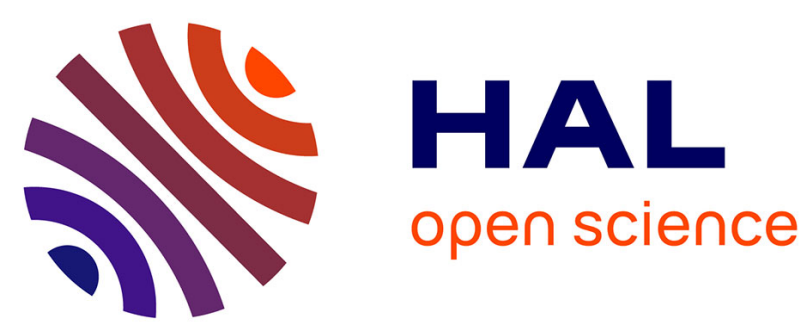

\title{
Dynamic line rating day-ahead forecasts - cost benefit based selection of the optimal quantile
}

\author{
Romain Dupin, Andrea Michiorri, Georges Kariniotakis
}

\section{To cite this version:}

Romain Dupin, Andrea Michiorri, Georges Kariniotakis. Dynamic line rating day-ahead forecasts cost benefit based selection of the optimal quantile. CIRED 2016 workshop - Electrical networks for society and people, CIREC - Centre International de Recherche sur l'Environnement et le Développement, Jun 2016, Helsinki, Finland. hal-01398440

\section{HAL Id: hal-01398440}

https://hal-mines-paristech.archives-ouvertes.fr/hal-01398440

Submitted on 17 Nov 2016

HAL is a multi-disciplinary open access archive for the deposit and dissemination of scientific research documents, whether they are published or not. The documents may come from teaching and research institutions in France or abroad, or from public or private research centers.
L'archive ouverte pluridisciplinaire HAL, est destinée au dépôt et à la diffusion de documents scientifiques de niveau recherche, publiés ou non, émanant des établissements d'enseignement et de recherche français ou étrangers, des laboratoires publics ou privés. 


\title{
DYNAMIC LINE RATING DAY-AHEAD FORECASTS - COST BENEFIT BASED SELECTION OF THE OPTIMAL QUANTILE
}

\author{
Romain DUPIN \\ Andrea MICHIORRI \\ Georges KARINIOTAKIS \\ romain.dupin@mines-paristech.fr andrea.michiorri@mines-paristech.fr georges.kariniotakis@mines-paristech.fr \\ MINES ParisTech, PSL - Research University, Centre PERSEE, France
}

\begin{abstract}
Dynamic Line Rating (DLR) is a promising field of research aiming to help network operators face challenges, such as increased penetration of renewable energies and peak electricity demand. Research on realtime overhead line ampacity estimation is currently advanced and, in the last few years, research has started to address medium-term DLR forecasting. The focus is on probabilistic forecasts, in order to select ratings associated with very low probability of occurrence. For this reason, 1\%-quantiles are usually selected. In this paper, the authors propose a methodology for selecting the most appropriate quantiles based on a cost-benefit analysis, considering both the economic benefits of an increased line ampacity and the costs associated with a DLR forecast that is higher than its observed value. The proposed methodology is evaluated using realistic weather data on a virtual line connecting Belgium and France.
\end{abstract}

\section{INTRODUCTION}

Nowadays, static seasonal ratings are applied to overhead lines in order to control statutory clearance. The corresponding current limit is traditionally calculated using methods defined in standards such those of IEEE [1] or CIGRE [2], and considering weather parameters defined as constant for a fixed period of time. This kind of rating is called Static Line Rating (SLR).

Due to its static nature, SLR is bound to be below the instantaneous rating most of the time, and thus restrictive weather characteristics are considered (i.e. low wind speed and high ambient temperature). This restrictive aspect may lead to congestion situations. To overcome this issue, an alternative way of defining the rating has emerged based on a dynamically modified ampacity that depends on real weather characteristics. This kind of rating is called Dynamic Line Rating (DLR). The development of real-time DLR tools is already advanced. A detailed state of the art on DLR technology is presented in [3]. Adopting DLR is expected to bring several benefits for network operators [4-6]. However, fully exploiting these benefits requires inputting information on DLR not only into real-time processes but also the various decision-making processes related to grid management for the forthcoming hours and days. This requires forecasting DLR for a future time period.

DLR forecasting has been addressed in the literature only recently. For medium-range forecasts (6-48 hours), models providing low-quantile forecasts were developed in [7-10]. Indeed in practical implementations, low quantile forecasts are preferred in order to avoid situations where the DLR forecasts are greater than the measurements. Infringing the thermal limit of an overhead line causes the line to sag more, which reduces the statutory clearance to the ground. However, note that in all the above works, the authors arbitrarily selected a quantile for their forecast, usually equal to $1 \%$. In this paper, a methodology is proposed for a dynamic selection of the quantile used for DLR day-ahead forecasts. This is compared with the choice of an arbitrarily chosen but fixed quantile. The proposed methodology is illustrated in the test case of a virtual line connecting Belgium and France, considering the local weather and the day-ahead and balancing markets in the two countries.

\section{METHODOLOGY}

\section{Optimal quantile selection}

A model providing probabilistic forecasts is a model that can calculate at time $t$, considering the information available, for a horizon $h$, a distribution function $\widehat{F}_{+h \mid t}$ for the possible values assumed by the parameter $\hat{Y}_{+h \mid t}$. To use the model in standard decision-making, it is often necessary to translate the probability density function calculated to a single value, for example by selecting the value $\hat{Y}_{t+h \mid t}^{\tau}$ corresponding to a specific quantile $\tau$.

The quantile $\tau$ can be arbitrarily selected, but it can also be the result of an optimisation, taking into account safety or financial aspects, as shown below. Such a selection is made considering that the forecast error $d^{\tau}{ }_{+h}=Y_{+h}-\hat{Y}_{t+h \mid t}^{\tau}$, has a cost per unit equal to $C_{t+h}^{+}$or $\mathrm{C}^{-}+h$ when its value is respectively positive or negative, as shown in Equation (1).

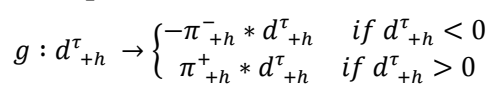

Considering the above costs, an optimal quantile can thus be selected through the minimization of the function (2), the optimization problem to be solved being described in (3).

$$
\begin{aligned}
E\left(\tau, \hat{F}_{t+h \mid t}\right) & =\int g\left(x-\hat{Y}_{+h \mid t}^{\tau}\right) * \hat{F}_{t+h \mid t}(x) d x \\
\tau_{+h}^{o p t} & =\operatorname{argmin}_{\tau}\left(E\left(\tau, \hat{F}_{t+h \mid t}\right)\right)
\end{aligned}
$$

An example of this approach is shown in [11], where the authors present a methodology according to which probabilistic wind power forecasts are used for trading wind generation in an electricity market with the goal of 
reducing imbalance penalties. The optimal forecast in this application is not necessarily the one that minimises the Mean Absolute Percentage Error (MAPE), but rather the one that minimises financial losses.

If the costs $\pi_{t+h}^{+}$and $\pi_{t+h}^{-}$are independent from $d_{t+h}^{\tau}$ and positive, the quantile minimizing this equation can be calculated analytically using formula in Equation (4):

$$
\tau_{+h}^{o p t}=\frac{\pi_{+h}^{+}}{\pi_{+h}^{+}+\pi_{+h}^{-}}
$$

\section{DLR Forecast optimised quantile}

The method described above is here adapted to DLR forecasting. We consider that a quantile forecast of the DLR, $\hat{Y}_{+h \mid t}^{\tau}$ can be calculated. In this case, both $\hat{Y}_{t+h \mid t}^{\tau}$ and $d^{\tau}{ }_{+h}$ are expressed in MVA. The DLR is considered as the thermal limit of the line, although this value should be reduced by taking into account other criteria due to grid limitations, for example: i) the limited size of balancing reserves on both sizes of the line, ii) the risk of removing $\mathrm{N}-1$ configurations in case a DLR forecast appears to be false, iii) the current stability limits, and iv) the thermal limits of other components, such as circuit breakers or transformers. However, for the sake of simplicity, these constraints are not considered in this study.

The following study is carried out for a line connecting two different electricity markets. The case study was designed to express simply the financial cost at both sides of the line due to errors related to DLR forecasting. As a reminder, as described in [12], a producer participating in an electricity market can go through four channels:

- The electricity futures market, on which long-term contracts on electricity bids are made.

- The day-ahead market, also called the spot market, on which a unique spot price is set depending on the proposed bids.

- The intraday market, on which the imbalances that occurred during the day can be corrected. This usually ends from 30 minutes to 2 hours before the time of delivery.

- Towards the end of the intraday market and the delivery time, possible imbalances are corrected during balancing by the transmission system operator. Positive and negative imbalances can have different costs.

The economic benefits associated with DLR forecasts are here considered to be dependent from the spot and imbalance costs. This involves assuming that the DLR forecast error has a different financial cost for positive and negative errors:

- If $d^{\tau}{ }_{+h}>0$, for a line connecting two areas with different spot electricity costs, $C^{1}$ and $C^{2}(€ / \mathrm{MW})$, the following cost is defined in Equation (5):

$$
\pi^{+}{ }_{+h}=-\left|C_{t}^{1}-C_{t}^{2}\right|
$$

This cost is linked to the fact that a better forecast would have increased the economic benefits relative to the use of the line. It is assumed that the increased rating of the line has no impact on $C_{t}{ }^{1}$ and $C_{t}{ }^{2}$.

- If $d^{\tau}{ }_{+h}<0$, the rating of the line is reduced to $Y_{t+h}$, and up and down reserves are activated in the two connected areas. If $C_{t}^{1}>C_{t}^{2}$, it causes a cost described in Equation (6). In the opposite case, the indexes need to be swapped:

$$
\pi_{+h}^{-}=\left(C_{t}^{\text {imbalance } 1^{-}}+C_{t}^{\text {imbalance } 2^{+}}\right)
$$

where $C^{\text {imbalance } 1^{-}}$is the regulation cost for negative imbalances in the first area. $C_{t}^{\text {imbalance } 2^{+}}$is the regulation cost for positive imbalances in the second area. It is supposed that both these parameters are independent from $d$.

At this point, considering (4), (5) and (6), the quantile optimising the financial benefits from DLR forecast can be calculated as in Equation (7):

$$
\tau_{\text {opt }}=\frac{\left|C^{1}-C_{t}{ }^{2}\right|}{\left|C^{1}-C_{t}{ }^{2}\right|+C_{t}^{\text {imbalance } 1^{-}}+C_{t}^{\text {imbalance } 2^{+}}}
$$

\section{CASE STUDY}

The test case considered here is a virtual line at the border of France and Belgium, connecting the two countries. It is located at the position $\left(3.125^{\circ} \mathrm{N}\right.$, $50.375^{\circ} \mathrm{E}$, only one point was considered in this study and not the whole length of the circuit), and was studied from 1st January 2014 to 31 st December 2015. We considered that the line is equipped with a $175 \mathrm{~mm}^{2}$ Lynx conductor.

The spot prices for France were provided by EPEX and those for Belgium by BELPEX. The balancing cost data were provided by the two national transmission system operators, RTE and Elia.

The DLR was calculated every 12 hours at the studied position, using reanalysis data provided by the European Center for Medium range Weather Forecasts (ECMWF) and the CIGRE standard method for calculating DLR [2]. The parameters used were:

- The $10 \mathrm{~m}$ East/West wind component $U f\left(\mathrm{~m} \cdot \mathrm{s}^{-1}\right)$.

- The $10 \mathrm{~m}$ North/South wind component $V f\left(\mathrm{~m} . \mathrm{s}^{-1}\right)$.

- The ambient temperature $T f\left({ }^{\circ} \mathrm{C}\right)$.

- The solar radiation $S$ set equal to $0 \mathrm{~W} \cdot \mathrm{m}^{-2}$.

The DLR forecasts were generated using Numerical Weather Predictions (NWP) provided by ECMWF for the same parameters, at 4 positions close to the studied point. The prediction horizon $h$ of the forecasts was equal to 24 hours. The forecasts were provided with a Quantile Regression Forest method (QRF) [13], which 
was trained with the data from 2014. Data from 2015 was used for the evaluation.

\section{RESULTS}

\section{DLR forecasts}

Day-ahead DLR forecasts were provided every 12 hours for the studied case, and the 99 percentiles associated with the forecasted DLR were calculated. As an example, the result obtained for one month is shown in Figure 1.

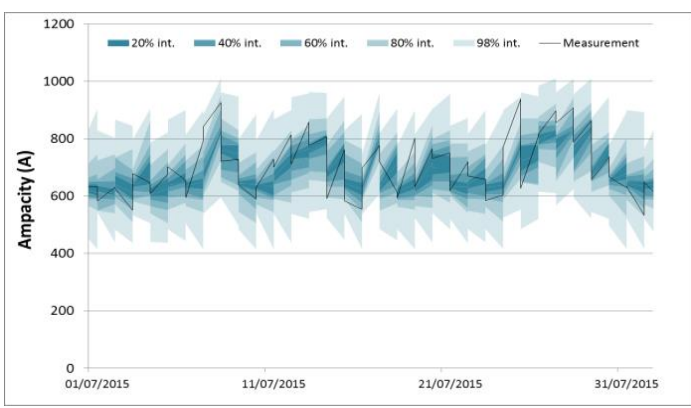

Figure 1. Consecutive day-ahead DLR forecasts from 01/07/2015 to $31 / 07 / 2015$.

Using the MAPE, we can compare the deterministic performances of the model with the ones of a persistence model, whereby the forecast made at $t$ for $t$ $+h$ is equal to the observation at $t$. A MAPE of $8.1 \%$ was calculated for the QRF, and of $11.4 \%$ for the persistence model.

\section{Optimised quantile}

Two methods were proposed for the quantile selection:

- An arbitrarily selected quantile $\tau_{\text {Arbitrary }}$.

- A dynamically modified quantile in accordance with the spot prices, selected using Equation (7). Depending on the values of the balancing costs used, two quantiles are calculated: $\tau_{\text {Spot }}$ with the mean balancing cost values of the same month during the previous year and $\tau_{\text {Spot,Balancing }}$ with observed balancing costs at time $t+h$.

For the first method, the economic benefits linked to the current flowing through the lines were calculated for various quantiles, from $1 \%$ to $100 \%$. As seen on Figure 2 , the benefits were optimised using the 14\%-quantile. This evaluation was made with the formula in Equation (8).

$$
\begin{gathered}
\mathrm{z}=\min \left(\hat{Y}_{t+h \mid t}^{\tau}, Y_{t+h}\right) *\left|C^{S P O T, 1}-C_{t}^{S P O T, 2}\right| \\
+\max \left(\hat{Y}_{t+h \mid t}^{\tau}-Y_{+h}, 0\right) *\left(C_{t}^{\text {imbalance } 1^{-}}+C_{t}^{\text {imbalance } 2^{+}}\right)
\end{gathered}
$$

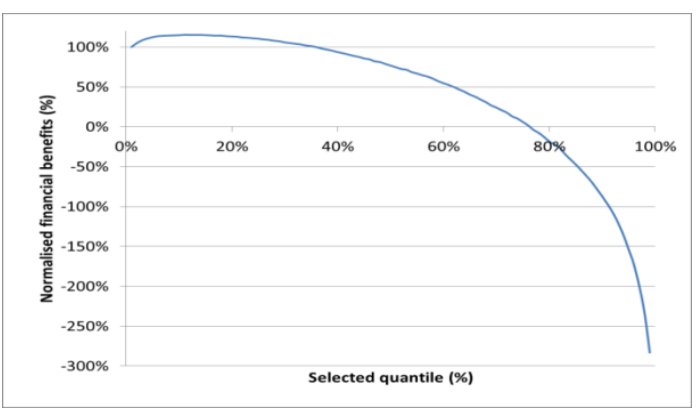

Figure 2. Benefits with an arbitrarily selected quantile

With the second method, the quantiles were calculated for 2015. The mean values for each month were provided in Table 1 .

\begin{tabular}{|l|c|c|}
\hline \multicolumn{1}{|c|}{ Parameter } & $\boldsymbol{\tau}_{\text {Spot }}$ & $\boldsymbol{\tau}_{\text {Spot,Balancing }}$ \\
\hline January & $2.5 \%$ & $2,6 \%$ \\
\hline February & $2.8 \%$ & $2,1 \%$ \\
\hline Mars & $4.1 \%$ & $3,8 \%$ \\
\hline April & $10.9 \%$ & $10,8 \%$ \\
\hline May & $12.9 \%$ & $14,7 \%$ \\
\hline June & $8.7 \%$ & $8,0 \%$ \\
\hline July & $7.4 \%$ & $8,0 \%$ \\
\hline August & $10.2 \%$ & $8,8 \%$ \\
\hline September & $15.2 \%$ & $18,2 \%$ \\
\hline October & $9.3 \%$ & $8,6 \%$ \\
\hline November & $3.9 \%$ & $4,9 \%$ \\
\hline December & $2.5 \%$ & $7,6 \%$ \\
\hline Mean value & $\mathbf{7 . 5 \%}$ & $\mathbf{8 . 2 \%}$ \\
\hline
\end{tabular}

Table 1. Mean optimal quantile selected during 2015.

In order to evaluate the financial benefit of the proposed approaches, 5 cases were tested and compared:

1. The $1 \%$-quantile DLR forecasts were used.

2. The 50\%-quantile DLR forecasts were used.

3. The $\tau_{\text {Arbitrary-quantile DLR forecasts were used. }}$ $\tau_{\text {Arbitrary }}$ is set as equal to $14 \%$.

4. The $\tau_{\text {Spot }}$-quantile DLR forecasts were used.

5. The $\tau_{\text {Spot,Balancing }}$-quantile DLR forecasts were used.

The benefits achieved during 2016 for the five cases were compared and the results are shown in Table 2 . The results were normalised with respect to the benefits achieved using an arbitrary $1 \%$ quantile.

\begin{tabular}{|c|c|}
\hline Case & Financial benefits respect to 1\% \\
\hline $1 \%$-forecasts & $100.0 \%$ \\
\hline $50 \%$-forecasts & $76.7 \%$ \\
\hline$\tau_{\text {Arbitrary }}$-forecasts & $115.1 \%$ \\
\hline$\tau_{\text {Spot }}$ forecasts & $115 \%$ \\
\hline$\tau_{\text {Spot,Balancing }}$ forecasts & $120.2 \%$ \\
\hline
\end{tabular}

Table 2. Normalized benefits for cases 1 to 5

The analysis of these results leads to the following considerations:

- Using an arbitrary 50\%-quantile of the forecasts was counter-productive.

- Increasing the chosen quantile from $1 \%$ to $14 \%$ increased the financial benefits by $15.1 \%$. 
- The choice of a variable optimal quantile taking into account spot prices and historical average balancing costs increased the benefits by $15.0 \%$. If real balancing costs at $t+h$ were taken into account, the benefits increased by $20.2 \%$.

\section{CONCLUSIONS}

The study presented a methodology for selecting the optimal quantile to determine a forecast DLR obtained using probabilistic methods. The methodology is based on a cost-benefit analysis and the aim is to use it, along with other criteria, to optimize day-ahead DLR forecasts.

The methodology proposed was tested on a test case describing a circuit on which DLR is applied, connecting two electricity market zones with different prices and balancing costs. This case is simple, and more complex situations should be studied further, but the observed results are promising and show potential benefits compared to the standard method using an arbitrarily selected low quantile.

It should be stressed that whilst the choice of the quantile is not influenced by the actual value of the rating, the financial benefit calculated is. This means that the results presented in Table 2 are more dependent on the data used, and in particular on the meteorological data used for rating calculations and forecasts, which are obtained from 12-hour reanalysis. The use of more precise historical weather data is expected to increase the precision of the forecasts and the results, but also to increase the variability of the rating. Moreover, this methodology based on a simple cost-benefit analysis does not take into account other DLR limitations, which should be included in further studies on adapting DLR forecasts to grid conditions. Other criteria, such as the costs associated with increased risks, could be considered when evaluating DLR forecast errors.

\section{ACKNOWLEDGMENT}

The following companies are acknowledged: ECMWF for access provided to the weather forecasts and reanalysis data; EPEX and BELPEX for the historical data on spot costs; RTE and Elia for the access provided to the historical data on balancing costs.

\section{REFERENCES}

[1] IEEE, "Standard for Calculating the CurrentTemperature Relationship of Bare Overhead Conductors," Transmission and distribution committee of the IEEE Power Engineering Society IEEE Std 738-1993, 1993.

[2] W. CIGRE, "12, Thermal Behaviour of Overhead Conductors, Tech," ed: Brochure, 2002.
[3] A. Michiorri, H.-M. Nguyen, S. Alessandrini, J. B. Bremnes, S. Dierer, E. Ferrero, et al., "Forecasting for dynamic line rating," Renewable and Sustainable Energy Reviews, vol. 52, pp. 1713-1730, 2015.

[4] M. Khaki, P. Musilek, J. Heckenbergerova, and D. Koval, "Electric power system cost/loss optimization using dynamic thermal rating and linear programming," in Electric Power and Energy Conference (EPEC), 2010 IEEE, 2010, pp. 1-6.

[5] C. J. Wallnerstrom, Y. Huang, and L. Soder, "Impact From Dynamic Line Rating on Wind Power Integration," Smart Grid, IEEE Transactions on, vol. 6, pp. 343-350, 2015.

[6] D. M. Greenwood and P. C. Taylor, "Investigating the impact of real-time thermal ratings on power network reliability," Power Systems, IEEE Transactions on, vol. 29, pp. 2460-2468, 2014.

[7] T. Ringelband, P. Schäfer, and A. Moser, "Probabilistic ampacity forecasting for overhead lines using weather forecast ensembles," Electrical Engineering, vol. 95, pp. 99-107, 2013.

[8] X. Sun, P. B. Luh, K. W. Cheung, and W. Guan, "Probabilistic forecasting of dynamic line rating for over-head transmission lines," in Power \& Energy Society General Meeting, 2015 IEEE, 2015, pp. 1-5.

[9] H.-M. Nguyen, J.-L. Lilien, and P. Schell, "Dynamic line rating and ampacity forecasting as the keys to optimise power line assets with the integration of res. The European project Twenties Demonstration inside Central Western Europe," in Electricity Distribution (CIRED 2013), 22nd International Conference and Exhibition on, 2013, pp. 1-4.

[10] "Final report on NETFLEX Demo," Twenties European Project,2013.

[11] P. Pinson, C. Chevallier, and G. N. Kariniotakis, "Trading wind generation from short-term probabilistic forecasts of wind power," Power Systems, IEEE Transactions on, vol. 22, pp. 1148-1156, 2007.

[12] R. Madlener and M. Kaufmann, "Power exchange spot market trading in Europe: theoretical considerations and empirical evidence," OSCOGEN (Optimisation of Cogeneration Systems in a Competitive Market Environment)-Project Deliverable, vol. 5, 2002.

[13] N. Meinshausen, "Quantile Regression Forest," Journal of Machine Learning Research, vol. 7, pp. 983-999, 2006. 